\title{
Autobiography of Fumio Hirata
}

Born in Nagasaki prefecture in Japan, right after the world war two, I grew up in a country side of Fukuoka prefecture, where I had my education until high school. After graduating from the high school, I entered Hokkaido University to study the natural science. The largest turning point as a student came to me when I knocked on the office door of Prof. Arakawa in 1969, who became my thesis advisor, in order to consult my study in the graduate course. He told me enthusiastically how "water" and "aqueous solutions" are important in science, how challenging it is to understand the problems experimentally as well as theoretically, and how interesting it is in the biological connections. In fact, at that time, the science related to water was about to flourish both in experiments and in theories accelerated in particular by the bio-scientific motivation such as the hydrophobic interactions. Both experimentalists and theorists were interested in the anomalous behavior which water exhibits in thermodynamic properties, such as the existence of the maximum density at 4 degree, and were trying to explain the behavior in terms of the structure of the liquid. Actually, the X-ray diffraction study of water, triggered by the landmark work by Bernal and Fowler in 1933, was producing the structural data which unequivocally indicate the existance of some tetrahedral coordination similar to that in ice. Concerning the theoretical studies, a couple of statistical mechanics theories, both based on a structural model of water, had been prevailing among the scientists in the field: one by Nemethy and Scheraga and the other by Pople. The model by Nemethy and Scheraga was a mixture model in which water is a mixture of nonbonded monomers and hydrogen-bonded clusters having one to four hydrogen bonds. Nemethy and Scheraga applied the free volume theory, a mean field theory based on the solid-like structure of liquids, to their model to reproduce the thermodynamic quantities and the cluster-size distribution. Pople proposed the bent-hydrogen-bond model of water structure, in which the thermal fluctuation of the ice-like structure in the tetrahedral coordination is represented by the "bending" of hydrogenbond from the linear configuration. Arakawa, who was basically an experimental chemist in the field of ultrasonic measurements of aqueous solutions, was also the first chemist in Japan to contribute to the statistical mechanics theory of liquids, especially of water. Arakawa had already proposed a couple of theories for water, both based on the two state model, hydrogen bonded or non-hydrogenbonded: one using the free volume theory, and the other employing the Brag-Williams-type theory of phase-transition. I believe that Arakawa's theories are the first treatment based on the two state model of water, although it has not been well appreciated in the recent booming of the two state model of water motivated by the finding of two phases in the amorphous ice, low density and high density. In any case, those theories which employ the structural model of water have only historical significance, since the essential theoretical problem to predict the structure of water from the first principle was untouched at that time.

Advised by Arakawa, I started my life as a baby scientist with an experimental study of the adiabatic compressibility concerning an aqueous solution of a series of the tetra-alkyl ammonium salts, and published my first paper. (1972) In the results, the temperature dependence of the compressibility reverses in between tetraethyl- and tetrapropyl-ammonium ions. The results were indicating some qualitative change in the hydration structure between those ammonium ions. The finding itself was not so big, but the study determined the direction of my future scientific activity. By struggling with the interpretation of the results of the experiment in order to extract microscopic view concerning what is going on in the hydration structure, I realized that there is no theory applicable to the process, and decided to change my research field to the statistical mechanics of liquids. During my Ph. D. thesis, I studied and developed three different theoretical treatments of water and aqueous solutions. One of those is the scaled particle theory, originated by Reiss, Frisch, and Lebowitz, and applied successfully to aqueous solutions by Pierotti. Applying the theory to the partial molar volume of ions in aqueous solutions, I proposed a new method to decompose the volume into the "intrinsic" and "electrostriction" terms, which had conventionally been done in empirical manner. (1973) The other theories that had a great impact on my thesis work were the analytical solutions of the Ornstein-Zernike (OZ) equations with the PY and MSA closures. After several months of struggling for understanding the theories, I picked up the analyt- 
ical solutions for the primitive model of electrolyte solutions obtained by Weisman and Lebowitz, and applied it to Friedman's model of the solutions. At that time, Friedman was developing a new theory of ionic solutions, in which the solvent induced interaction between two ions in water is modeled by the so-called "Gurney terms". It was the first serious attempt to put forward the electrolyte solution theory beyond the "primitive model". I combined the Freidman model and the Weisman-Lebowitz solution into the Zwanzig-type perturbation theory of liquids to describe the realistic aqueous solutions of electrolytes: the primitive model and the Gurney term as the reference and the perturbation, respectively. (1975) The theory was successful in the sense that it could produce the Gurney terms which are consistent with the empirical models of ion hydration proposed by Frank and Wen as well as Samoilov in 1957. However, the Gurney terms are essentially phenomenological, and this did not give a complete satisfaction to me. I then started to study formalisms which might be capable of being applied to water, and found the seminar papers written by Chandler, Andersen, and Weeks. The papers were proposing two models of molecular liquids, which are closely related to each other, namely the reference interaction-site model (RISM), and the embedded site model (ESM). I naturally picked up ESM, because it employed the analytical solution of the PY equation for the hard-sphere system as the reference for the diagrammatic perturbation expansion of the site-site pair correlation functions of molecular liquids. Needless to say, I was already familiar with the analytical solutions, while at that time I did not have numerical techniques sufficient to solve the RISM integral equation. In order to apply the ESM theory to the structure of water, I made up a model of water, which is similar to that proposed by Ben-Naim. The hydrogen-bond is represented by a square-well-type interaction between the interaction sites which are located symmetrically at the vertices of a tetrahedron embedded in a hard-sphere. The electrostatic interaction was taken into account by placing an ideal dipole at the center of spheres. Regarding the hydrogen-bond as well as the dipole-dipole interaction as a perturbation to the hard-sphere interaction, I applied the ESM theory to the model water system. (1977) The theory and the model qualitatively reproduced the two characteristics of water structure, reflected in the oxygen-oxygen radial distribution functions (RDF). It has been well regarded since the paper by Bernal and Fowler that the first peak of RDF is considerably narrower than that in simple liquids, and that the second peak appears at the position similar to that in ice, both being manifestations of the ice-like tetrahedral coordination of the hydrogen-bond network in water. Although the theory did not attract too much attention, it probably was the first attempt to "qualitatively" reproduce the "signature" of water structure from the first principle.

Having completed the Doctor thesis, I started the postdoctoral work at Prof. H. Friedman's lab in 1978 in the State University of New York at Stony Brook. During the one year stay in Stony Brook, I was able to publish only one paper with Friedman, which concerns the spin relaxation in solution. (1980) However, I was able to learn from Friedman so many things which became vital to my scientific career. Among those is the numerical treatment of the OZ-type integral equations. Especially, the recursive solution of the renormalized OZ equation for the Coulomb interaction was the one which gave me the idea for the extended RISM theory later on. The other gene I inherited from Friedman concerns the dynamics of liquids. Friedman is best known far his contribution to the theory of electrolyte solutions. However, his contribution to the dynamics of liquids and solutions is at least as big, and the spin relaxation in ionic solutions was his latest concern in that category. In the work on the spin relaxation, I acquired the knowledge about how to treat the liquid dynamics on the research level rather than on the textbook level. When about a year past at Stony Brook, Prof. P. Rossky, who then was also a postdoc of Friedman's group, was offered a tenure track position in the University of Texas, and asked me to join his group as a postdoc. I thought it would be a great opportunity to study the molecular dynamics simulation from the scientist who was among the leaders in that field, and decided to go to Austin. At UT, we promptly published two papers concerning the MD simulation of water and aqueous solutions based on the idea which Rossky had incubated. One of those was the MD study to realize the "V-structure" of water proposed by Kauzmann in his famous book. (1981) Having completed those papers, we were discussing our next work. Rossky was thinking to elaborate the Pratt-Chandler theory of the hydrophobic interaction by replacing the experimental RDFs with those from the MD simulation. On the other hand, I was 
thinking of renormalizing the RISM theory for the Coulomb interactions to be applicable to polar liquids including water. After listening to my idea, Rossky almost immediately said "that is much more challenging. Let's do it". After almost one year of struggling, we completed the theoretical formulation as well as the numerical calculation for the extended RISM theory. The work was published in the three monumental papers. (1981-1983) After two years of stay in Texas, I decided to go back to Japan in order to release the stress from insecurity. I got a job in a software house, called NASAC, featuring the safety analysis of nuclear power plant. The job itself was nothing related to scientific research, but it brought new possibilities to me. During the last two years of the position in the company, I was hired by Fujitsu, the computer giant in Japan, for participating in the project of making the computer graphics movie entitled "THE UNIVERSE", which was exhibited in the Tsukuba Science Exposition held in 1985. I was expected to play dual roles in the project: one to perform the molecular dynamics simulations of water, ice, protein, and DNA, and the other to mediate between the Japanese workers and the American CG scientists. When I was making programs to perform the simulation for the biomolecules, I became interested in the bio-science which had never drawn my attention before. Although almost the entire family except my wife was against my idea to go abroad, I decided to go to US again to do research in the biophysical science. In Rutgers together with Prof. R. Levy, I turned my steering toward the biooriented science as I had intended. The atmosphere in Rutgers was almost ideal for me to pursue the research along such a direction. The university was putting a lot of effort to building up the bio-scientific research. The chemistry department had a large and well-reputed theoretical group including R. Levy, G. Manning, W. Orson, from whom I was able to learn a lot about biophysics. Two big names in the statistical mechanics of liquids, J. Lebowitz and M. Wertheim, whose paper gave a great influence on my earlier work, were also in the same campus. The biggest contribution by myself in Rutgers was to participate in the project to build a simulation program entitled "IMPACT", which facilitates the simulation of biomolecules, protein and DNA, in water and aqueous solution. At that time, the protein simulation originated and driven by M. Karplus' group was expanding in the world, and was entering the second phase of the scientific achievement, which was to take the realistic environment of aqueous solutions into account. Levy was also struggling to incorporate "water" in his protein simulation program. So, it was just the right time when I took the office in Levy's lab, as a "water expert". After making a long and extensive survey concerning the available simulation program, we decided to make a new program from scratch. For the entire period of four years stay in Rutgers, I was participating in the project without writing a single paper using that program. However, the experience was impeccable for me to build my background on the biophysics, which became vital in my later work. It was the extended RISM theory that let me produce scientific papers during that time. In Rutgers, I started to apply the extended RISM theory to realistic problems in aqueous solutions, including biophysical phenomena. One of those concerns the hydration free energy of ions. (1987) I calculated the free energy and entropy of a series of alkali and halide ions to prove that the size dependency of those quantities falls on entirely different curves as opposed to the prediction from the Born formula of ion hydration, and that the difference is attributed to the asymmetric charge distribution in water molecules. The other contribution I made in the statistical mechanics of liquids was the polymer RISM theory. Unlike today, the numerical algorithm of solving the RISM equation and the computer machine power were so poor then that the conventional RISM theory could not be applied directly to biomolecules, protein and DNA, having many interaction sites. Considering such a situation, I proposed a RISM theory which uses the periodicity of the interaction sites in a polymer, and applied the theory to the salt induced transition of DNA from the B to Z forms. (1989) Although the application was largely limited to particular polymers in which one can count on a periodicity, the theory was the first molecular theory of polymer solvation. That was the time I was offered an associate professor position at the chemistry department of Kyoto university in 1989, where they were searching for a theorist in the field of biophysics.

In Kyoto university, I started two new problems in the chemical physics, along with the molecular simulation of biomolecules. The first one is the electronic structure of a solvated molecule in solutions in collaboration with Tenno, then a graduate student, and with Prof. Kato. (1993) 
Motivated by Kuki, then a professor in Cornel University, who informed me on the contemporary booming of the generalized Born formalism to treat the solvent effect on the electronic structure, I came up with the idea of hybridizing the extended RISM theory with the ab initio molecular orbital method. The new theory named "RISM-SCF" has found many applications in chemistry, and has become a standard in the filed. The "solvated Fock operator" introduced rather heuristically in the original paper has been put on a solid theoretical foundation due to the variational principle, by Sato, then a graduate student. The other theory I started in my period of Kyoto university is the dynamics of molecular liquids and solutions. The motivations were brought from two experimental fields. One of those came from the field of the dynamics Stokes shift, especially from Okada, then a professor in Osaka university, who introduced me the fascinating phenomena of the ultra-fast spectroscopy. The other experiment which excited me was the solitary molecular dynamics in solution based on the NMR relaxation, measured by Prof. Nakahara and his coworkers. It was a natural direction to employ the generalized Langevin theory for the purpose of building a theory of liquid dynamics from the time evolution in the phase space, and many scientists were trying to make a theory for the dynamics of liquids from such a viewpoint. However, most of those theories had ended up with the phenomenological expression for the dielectric relaxation founded by P. Debye in early last century. The reason was obvious. There was no molecular theory available at that time for the dynamics of liquid consisting of polyatomic molecules, including water. Urged by that situation, I formulated a new theory of liquid dynamics combining the RISM theory with the generalized Langevin equation (GLE), and calculated the van Hove space time correlation function of water for the first time. (1992) Although the success was limited because I employed the overdumped regime to introduce one phenomenological parameter, or the diffusion constant, all the important physics to make a more elaborate theory were already there. The most important element was a conceptual model regarding the dynamics of polyatomic molecules. The traditional theory of the liquid dynamics employs the spherical harmonics to describe the rotational dynamics. Such a description, however, diverges quickly when the asphericity of molecules becomes large. I constructed a new model which sees the dynamics of a molecule as a correlated translational motion of atoms composing the molecule: if two atoms in a molecule move in the same direction, it results in the translational motion, while if they move in the opposite directions, it gives rise to the rotational motion. The idea was realized by choosing the density field of atoms as dynamic variables, which enables one to incorporate the density pair correlation functions from RISM into the GLE formalism. During my occupation in Kyoto university, I added three other contributions to the chemical physics of liquids together with my collaborators. One of those concerns the molecular dynamics simulation of a peptide in water. Kitao, Hayward, Go, then a professor in Kyoto University, and I contributed to propose the principal component analysis to extract the "effective modes" of protein in water, which is now a standard tool to analyse the trajectory of macromolecules in solution. (1991) In the study, "IMPACT" was used to produce the trajectory of the peptide as well as water molecules. One of the other contributions concerns the scaled particle theory which is originally meant for a system consisting of hard spheres. Irisa, Nagayama, and I extend the scaled particle theory to the system in which solute consists of many atoms, such as protein.(1993) The other contribution, which was made by Chong, Miura, Basu, and I, is to use the RISM theory to realize the non-equilibrium free energy surface of the electron transfer reactions based on the same concept proposed by R. Marcus. (1994)

My scientific activities became in full throttle at the new environment in the Institute for Molecular Science in Okazaki, where distinguished scientists in the field of chemistry and physics are put together. My contributions in Okazaki are categorized into four fields, in each of which I constructed new theories and methodologies in the chemical physics of condensed matter: (1) the electronic structure and chemical reactions in solution, (2) the solvation thermodynamics and folding of protein, (3) the dynamics of liquids, and (4) the interfacial liquid. My collaborators applied the RISM-SCF theory to a variety of chemistries in solutions no matter what it is if the electronic structure change is involved: the organic reactions including acid-base, Sn2, Diels-Alder, redox, and the solvatochromism. The applications are quite successful, enough to be appreciated as a standard, in interpreting the physicochemical processes produced by the interplay between 
the electronic structure of solute and the many-body effect due to solvent. In IMS, I had a good fortune to challenge the protein folding, the most highlighted problem in the biophysics during the last few decades, which is to find a native structure of protein provided the primary sequence of amino acids is available. There are two keys to solve the puzzle proposed by Anfinsen in the last century. One of them is to find a simulation algorithm which can sample the conformational space of protein without having the non-ergordic trap in a local energy minimum. The other is to calculate the free energy surface of the protein, which accounts properly for the solvation free energy. Before coming to IMS, I was warming an idea of protein folding, which is to combine the RISM theory and a simulation algorithm to solve the two problems stated above. The idea was realized by the encounter of three people, Profs Okamoto, Kinoshita, and myself. Kinoshita was introduced to me by Prof. G. Patey in the JRDC Forum for "Water - Structure and Properties" (organized by Ohtaki) held in Kyoto in 1994. Kinoshita was well-armed by the numerical solution technique of the OZ type integral equations. The technique was impeccable for the combined algorithm, because it requires the fast and stable solutions of the RISM equation in order to evaluate the solvation free energy for a huge number of conformations of protein produced by the simulation. Okamoto, who was developing the generalized ensemble algorithm for sampling the conformation space, was already in IMS one year before I took the office, and was struggling to get the solvent effect in his algorithm. The combined algorithm was applied to a small peptide called "enkephalin" which is a "pet" molecule of the protein folding community. The new method reproduced successfully the structure of the molecule in water, found using the 2D-NMR technique. (1998) In the dynamics of liquids, my idea sprouted in Kyoto was further extended and generalized by the collaborators, Chong, Yamaguchi, and Kobryn. Chong and I introduced the mode coupling formalism into the site-site generalized Langevin theory of molecular liquids, and identified the translational and rotational dynamics of molecules as the acoustic and optical modes, respectively, based on the concept of the collective excitation. (1998) They further applied the concept to the dynamics of an ion in polar liquid. The problem had been challenged by M. Born, R. Zwanzig, J. Hubbard, L. Onsager, more recently by P. Wolynes, employing essentially phenomenological concepts such as the Stokes-Einstein law, the dielectric friction, and the "solvent berg" model. The question to be answered is why the dynamics of ions exhibits the size dependency opposite to the Stokes law when the size is small. Chong and I described the friction exerted on an ion in terms of the response of the collective dynamics of the solvent to the ion-solvent interaction. The anti-Stokes-law like behavior in the small ion-size region was reproduced by the theory, and was identified as the response of the solvent optical mode to the Coulomb interaction. Liquids at boundary with the other phases of matter are the phenomena I have tried to describe in terms of the statistical mechanics, in collaboration with Kovalenko and other people. Of course, the scientific challenges have never been made just by applying the conventional RISM theory to the problems. In fact, the original RISM theory with the conventional closure relations, PY, HNC, and MSA, exposes its weakness in some applications. One of the weakness inherent in the RISM theory concerns the intra-molecular correlation functions, which is not always strong enough to exclude solvent molecules from the core region of a solute molecule, say, protein. The problem shows up when one tries to calculate the radial distribution functions of water around atoms buried deep inside a globular protein; the radial distribution function quickly decays to unity, or the bulk value, although there should not be any water molecules around. A phenomenological manifestation of the trouble is seen in the partial molar volume of globular protein; when the volume is plotted against the size of protein, it exhibits unphysical behavior. The illness was cured by the 3D-RISM theory in which the average overorientation is taken only for solvent molecules. (1998) The method combined with the Kohn-Sham type density functional theory was first applied to the electrodewater interface in order to consistently describe the electronic structure of the metal and the solvent distribution, pretty much in the same spirit as the RISM-SCF theory. (1999) The power of the 3D-RISM theory has been fully demonstrated in the solvation structure and thermodynamics of protein. The partial molar volumes of proteins in aqueous solutions calculated by Imai, Kovalenko, and I have exhibited a quantitative agreement with the corresponding experimental results (2004). These turn out to be the first quantitative results obtained for the thermodynamics of protein 
entirely from the statistical mechanics theory. It was a great accomplishment by itself in the sense that it gave a big confidence to the 3D-RISM to explore the stability of protein in solutions. However, it was only a prelude to a discovery which will give even bigger impact on the science. When Imai and his student, Hiraoka in Ritsumeikan University, were analysing the 3D-distribution of water around hen egg-white lysozyme, they found conspicuous peaks inside small cavities in the protein, which no doubt reveal the water molecules trapped inside the macromolecule. In fact, the number of water molecules and the positions inside a cavity coincide with those found by the Xray crystallography (2005). This implies that the 3D-RISM is capable of "detecting" the molecules "recognized" by protein, or the host molecule. In other words, the 3D-RISM theory can be applied to the "molecular recognition" with which almost all processes in bio-systems to maintain their life are concerned: the information transmission, the enzymatic reactions, drug binding, etc. For the enzymatic reaction to occur, substrate molecules should be first accommodated by the protein in its reaction pocket to form the so-called "ES-complex". For an ion to permeate through the cell membrane, it should first be accommodated inside the pore of the ion channel. Such a process will never be described by the continuum model, because the model does not have the concept of the "molecule" to be recognized. The molecular simulation can be applied to the process in some limited case, but it will never be predictive. Why is it so? This is because the molecular simulation is designed essentially for sampling a limited area in the phase space. The molecular recognition, which is a rare event, requires the sampling of the area in the phase space which the molecular simulation cannot access. At best, the method is capable of calculating the free energy change from bulk to the host cavity using the thermodynamic perturbation technique. However, the method requires in advance the experimental information regarding the position and the number of ligands inside the host cavity as well as the number of water molecules to be replaced. Unfortunately, the information is the one which should be predicted by the theory. The reason why the 3DRISM theory was capable of predicting the molecular recognition is because it samples over the entire phase space by the configuration integral which of course includes that corresponding to the extreme rare event. Due to all stated above, the paper will be considered as the monumental work not only in the field of the physical science, but also in the field of the life sciences. The other weakness of the conventional RISM theory is displayed in low density region of liquids, or in dilute concentration region of liquid mixtures. The problem has prevented the RISM theory from being applied to the intriguing phenomena like the water-alcohol mixture, the vapor-liquid phase transition, and the liquid-liquid interface, where the pair correlation functions in the low density regime are inevitably involved. Kovalenko and I installed a new closure relation, the KH closure, which turns out to be a remedy to the ill-behavior in the low density fluids. The RISM-KH theory has been applied to explore a variety of phase behaviors concerning the low density regime. One of them concerns the vapor-liquid phase transition of water (2001). Since the land-mark paper published by van der Waals, a lot of effort has been devoted to put the phenomenological theory on the foundation of statistical mechanics. However, no serious attempts have been made to produce the phase diagram for realistic molecular fluids. The theory by Kovalenko and me turns out to be the first statistical mechanics theory for the vapor-liquid phase transition of water. Another interesting application of the RISM-KH theory has been made to the alcohol-water mixture. The wateralcohol mixtures exhibit an enormous variety from complete mixing to phase separation depending on thermodynamic conditions, and on the size and structure of the alkyl groups. It has been a long-standing puzzle how the thermodynamic behavior is related to the structure of the solution. Kovalenko, Yoshida, Yamaguchi, and I have calculated the radial distribution functions between all the pairs of atoms in the t-butanol-water mixture changing the concentration, from which we were able to extract an unambiguous solution structure (2002). According to their results, in the infinite dilution of t-butanol in water, the alcohol molecule is incorporated in the hydrogen-bond network of water forming the bonds with the solvent. At the low but finite concentration of alcohol, the molecules again participate in the hydrogen-bond network with making their alkyl groups into face-to-face contact. The solution structure turns out to explain the peculiar behavior which the mixture exhibits in the thermodynamic quantity. The isothermal compressibility of the mixture plotted against alcohol concentration passes through a minimum. Omelyan, Kovalenko, and I not 
only reproduced the experimental behavior with the RISM-KH theory, but also succeeded in giving a structural explanation to the phenomenon: alkyl groups of alcohols tend to "fill" the space in the hydrogen-bond network of water with the mechanism stated above, which reduces the compressible space of the solvent (2003). The boundary between two fluid phases is another important challenge to the RISM-KH theory. Omelyan, Kovalenko, and I succeeded in reproducing the density profile of the boundary between the gas and liquid phases as well as the coexisting curve for a LennardJones fluid supplemented with the Lovett-Mou-Buff-Wertheim (LMBW) theory for inhomogeneous liquids. (2004) To my knowledge, this is the first theory to reproduce the density profile of the boundary between two fluid phases entirely from the first principle. However, it was not the whole story. The real surprise to us was the behavior of the pair correlation functions at the boundary. The function in the plane parallel to the fluid boundary exhibits an extremely long-lasting tail, which is akin to that seen in the critical point. By examining the critical exponent we managed to prove that in fact this is a manifestation of the critical behavior. The similar behavior is observed in the liquid-liquid interface. Kovalenko and I calculated the concentration profile of the boundary between the two solution phases of a methanol-n-hexane mixture. (2005) The results exhibit a clear indication of the critical behavior, which has been conventionally called "capillary wave".

My scientific career has been devoted to reberating the statistical mechanics from the narrow cage of "physics" into the real world of chemistry and biology, so that the theory demonstrates its real power in exploring the nature. The phenomenological models such as the continuum dielectrics introduced into chemistry in the early last century by the giants in the science will lose the significance of their existence, and will sooner or later disappear from the scene of molecular science. The statistical mechanics of molecular liquids, or the RISM theory, and the increasing computer power will help them fall into the eternal sleep.

Having this opportunity, I would like to express my deep gratitude to my thesis advisor, Prof. Arakawa, for guiding me to the statistical mechanics of liquids, and to Profs. Friedman, Rossky, and Levy for providing me the research positions in the United States. I am also grateful to Prof. Go who helped me in coming back to work in Japan. I thank all my collaborators who have struggled with me to create the liquid state theory in chemistry. 


\section{Publications of Fumio Hirata}

1. Hirata F., Arakawa K. Ultrasonic Study of Solute-Solvent Interactions in Aqueous Solutions of Tetraalkylammonium Salts. Bull. Chem. Soc. Japan, 1972, 45, 2715.

2. Hirata F., Arakawa K. Molar Volume of Ions. Bull. Chem. Soc. Japan, 1973, 46, 3367.

3. Hirata F., Arakawa K. The Computation of the Thermodynamic Properties of Aqueous Electrolyte Solutions by Means of the Perturbation Theory of Fluid. Bull. Chem. Soc. Japan, 1975, 48, 2139.

4. Hirata F., Statistical Mechanical Calculation of the Radial Distribution Function for a Water-like Fluid, Bull. Chem. Soc. Japan, 1977, 50, 1032.

5. Hirata F., Friedman H. L., Holtz H., Hertz G. NMR Relaxation Study of Li+-Ni2+ Interaction in Aqueous Solution. J. Chem. Phys., 1980, 73, 6031.

6. Hirata F., Rossky P.J. On the Interpretation of Solute Induced Solvent Structure. J. Chem. Phys., 1981, 74, 6031 .

7. Hirata F., Rossky P.J. A Realization of 'V-structure' in Water. J. Chem. Phys., 1981, 74, 6867.

8. Hirata F., Rossky P.J. An Extended RISM Equation for Molecular Polar Liquids. Chem. Phys. Lett., 1981, 83, 329

9. Hirata F., Pettitt B.M., Rossky P.J. Application of an Extended RISM Equation to Dipolar and Quadrupolar Fluids. J. Chem. Phys., 1982, 77, 509.

10. Rossky P.J., Hirata F. The contribution of High Frequency Intermolecular Motions to the Structure of Liquid Water, in Molecular based Study of Fluids, ACS advances in Chemistry Series. American Chemical Society, Washington D.C., 1983, 281-296.

11. Hirata F., Rossky P.J., Pettitt B.M. The Interionic Potential of Mean Force in a Molecular Polar Solvent from an Extended RISM Equation. J. Chem. Phys., 1983, 78, 4133.

12. Gallion S. L., Levy R. M., Weiner P. K., Hirata F. Implementation of a Macromolecular Mechanics Program on a CYBER 205 Supercomputer. Computers \& Chemistry, 1986, 10, 165.

13. Hirata F., Levy R. M. Ionic Association in Methanol and Related Solvents: An Extended RISM Analysis. J. Phys. Chem., 1987, 91, 4788.

14. Hirata F., Levy R. A New RISM Integral Equation for Polymers Interacting with Solvent. Chem. Phys. Lett., 1987, 136, 267.

15. Using Computer Simulations to Probe the Structure and Dynamics of Biopolymers, Hirata F., Levy R., Kim K., Zhang P. in Supercomputer Research in Chemistry and Chemical Engineering, ACS Symposium Series, 353, American Chemical Society, Washington D. C., 1987, 82-105.

16.Bassolino D., Hirata F., Kitchen D.B., Kominos D., Pardi A.,Levy R.M. Determination of Protein Structures in Solution Using NMR Data and IMPACT. International Journal of Supercomputer Applications, 1988, 2, 41.

16. Hirata F., Levy R. Viewing The Born Model for Ion Hydration through A Microscope. International Journal of Quantum Chemistry: Quantum Biologie Symposium, 1988, 15, 179.

17. Hirata F., Levy R. Salt-Induced Conformational Change in DNA: Analysis Using the Polymer RISM Theory. J. Phys. Chem., 1989, 93, 479.

18. Kitchen D.B., Hirata F., Kofke D.A., Westbrook D., Levy R.M. Conserving Energy During Molecular Dynamics Simulations of Water, Proteins, and Proteins in Water. J. Comp. Chem., 1990, 11, 1169.

19. Kitchen D.B., Hirata F., Levy R.M.Conserving Energy in Molecular Dynamics Simulations of Proteins in Water. Theoretical Chemistry \& Molecular Biophysics, Eds., D. L. Beveridge \& R. Lavery, Adenine Press, 1990, 77.

20. Kitao A., Hirata F., Go N. The Effect of Solvent on the Conformation and the Collective Motions of Protein: Normal Mode Analysis and Molecular Dynamics Simulations of Melittin in Water and in Vacuum. Chem. Phys., 1991, 158, 447.

21. Hirata F. Interaction-site Representation of The Smoluchowski-Vlasov Equation: The Space-Time Correaltion Functions in a Molecular Liquid. J. Chem. Phys., 1992, 15, 4619.

22. Irisa M., Nagayama K., Hirata F. Extended Scaled Particle Theory for Dilute Solutions of Arbitrary Shaped Solute: An Application to Solvation Free Energies of Hydrocarbons. Chem. Phys. Letter, 1993, 207, 430 . 
23. Hayward S., Kitao A., Hirata F., Go N. Effect of Solvent on Collective Motions in BPTI. J. Mol. Biol., 1993, 234, 1207.

24. Kitao A., Hirata F., Go N. The Effect of Solvent on the Conformation and the Collective Motions of Protein: II. Structure of Hydration in Melittin. J. Phys. Chem., 1993, 97, 10223.

25. Kitao A., Hirata F., Go N. The Effect of Solvent on The Conformation and The Collective Motions of Protein: III.Free Energy Analysis by The Extended RISM Theory. J. Phys. Chem., 1993, 97, 10231.

26. Tenno S., Hirata F., Kato S. A Hybrid Approach for the Solvent Effect on the Electronic Structure of a Solute Sased on the RISM and Hartree-Fock Equations. Chem. Phys. Lett., 1993, 214, 391.

27. Raineri F.O., Resat H., Pern B., Hirata F. Hriedman H.L. A Molecular Theory of Solvation Dynamics. J. Chem. Phys., 1994, 100, 1477.

28. Tenno S., Hirata F., Kato S. Reference Interaction Site Model Self-Consistent Field Study for Solvation Effect on Carbonyl Compounds in Aqueous Solution. J. Chem. Phys., 1994, 100, 7443.

29. Basu G., Kitao A., Hirata F., Go N. A Collective Motion Description of the 310-/a-Helix Transition: Implications for a Natural Reaction Coordinate. J. Am. Chem. Soc., 1994, 116, 6307.

30. Miura S., Hirata F. Temperature Dependence of the Stability of a Hydrated Electron: An Integral Equation Study. J. Phys. Chem., 1994, 98, 9649.

31. Friedman H.L., Raineri F., Hirata F., Pern B. Surrogate Hamiltonian Description of Solvation Dynamics Site-Number Density and Polarization Charge Density Formulation. J. Stat. Phys., 1995, 78, 239 .

32. Kawata M., Tenno S., Kato S., Hirata F. Irregular Order in Basicities of Methylamines in Aqueous Solutions: A RISM-SCF Study. J. Am. Chem. Soc., 1995, 117, 1638.

33. Chong S.-H., Miura S., Basu G., Hirata F. Molecular Theory for the Non- Equilibrium Free Energy Profile in Electron Transfer Reaction. J. Phys. Chem., 1995, 99, 10526.

34. Kawata M., Tenno S., Kato S., Hirata F. The Solvation Effect on the Acidities of Haloacetic Acids in Aqueous Solutions: A RISM-SCF Study. Chem. Phys. Letter, 1995, 199, 240.

35. Irisa M., Takahashi T., Nagayama K., Hirata F. Solvation Free Energies of Non-polar and Polar Solutes Reproduced by the Combination of the Extended Scaled Particle Theory and the PoissonBoltzmann Equation. Mol. Phys., 1995,85, 1227.

36. Hirata F., Munakata T., Raineri F., Friedman H.L. An Interaction-Site Representation of the Dynamic Structure Factor of Liquid and Solvation Dynamics. J. Mol. Liquids, 1995, 65-66, 15.

37. Chong S-H., Hirata F. Non-linear Effects in the Free Energy Profile of Electron Transfer Reaction: an Integral Equation Approach. J. Mol. Liquids, 1995, 65-66, 345.

38. Irisa M., Takahashi T., Hirata F., Yanagida T. Solvation Free Energy of Protein Reproduced by the Combination of the Extended Scaled Particle Theory and the Poisson-Boltzmann Equation. J. Mol. Liquids, 1995, 65-66, 381.

39. Chong S.-H., Hirata F. The Free Energy Profiles for the Electron Transfer Reactions Calculated from the Integral Equation Method of Liquids. Molecular Simulation, 1996, 16, 3.

40. Kawata M., Ten-no S., Kato S., Hirata F. Solvent Effect on Acidity: A Hybrid Approach Based on the RISM and Hartree-Fock Equation. J. Phys. Chem., 1996, 100, 1111.

41. Kawata M., Ten-no S., Kato S., Hirata F.. Theoretical Study of the Basicities of Methylamines in Aqueous Solutions: A RISM-SCF Calculation of Solvation Theromodynamics. Chem. Phys., 1996, 203, 53 .

42. Kinoshita M., Hirata F. Application of the Reference Interaction Site Model Theory to Analysis on Surface-Induced Structure of Water. J. Chem. Phys., 1996, 104, 8807.

43. Ueoka R., Matsumoto Y., Goto K., Ito T., Mori S., Matsumoto Y., Sakoguchi A., Ihara Y., Hirata F. A Remarkably Enhanced Diastereoselectivity for the Hydrolysis of Dipeptide Esters Responding to $\mathrm{pH}$ and Temperature in Buffer Solutions. Tetrahedron letters, 1996, 37, 3461.

44. Sato H., Hirata F., Kato S. Analytical Energy Gradient for the Reference Interaction Site Model Multiconfigurational Self-Consistent-Field Method: Application to 1,2-Difluoroethylene in Aqueous Solution. J. Chem. Phys., 1996, 105, 1546. 
45. Yoshida S., Hirata F., Munakata T. Shear-Induced Distortion of Intramolecular and Intermolecular Correlations in Liquids: Time-Dependent Density-Functional Theory. Phys. Rev. E., 1996, 52, 1763.

46. Munakata T., Yoshida S., Hirata F. Statistical Mechanics of Deformable Liquids: Thermal Expansion and Isomerization of Diatomic Molecules. Phys. Rev. E., 1996, 54, 3687.

47. Chong S.-H., Hirata F. Nonlinear Electrical Potential Fluctuations of Solvent Around Solutes: An Integral Equation Study, J. Chem. Phys., 1997, 106, 5225.

48. Chong S.-H., Hirata F. Ion Hydration: Thermodynamic and Structural Analysis with an Integral Equation Theory of Liquids. J. Phys. Chem. B, 1997, 101, No. 106, 3209.

49. Kinoshita M., Hirata F. Analysis of Salt Effects on Solubility of Noble Gases in Water Using the Reference Interaction Site Model Theory. J. Chem. Phys., 1997, 106, No. 12, 5202.

50. Maw S., Sato H., Tenno S., Hirata F. Ab Initio Study of Water: Self-Consistent Determination of Electronic Structure and Liquid State Properties, Chem. Phys. Lett., 1997, 276, No. 1-2, 20.

51. Kinoshita M., Okamoto Y., Hirata F. Calculation of Hydration Free Energy for a Solute with Many Atomic Sites Using the RISM Theory: A Robust and Efficient Algorithm. J. Comp. Chem., 1997, 18, No. 10, 1320.

52. Kinoshita M., Okamoto Y., Hirata F. Solvation Structure and Stability of Peptides in Aqueous Solutions Analyzed by the Reference Interaction Site Model Theory. J. Chem. Phys., 1997, 107, No. 5, 1586.

53. Chong S.-H., Hirata F. An Integral Equation Study for Non-Linear Electrical Potential Fluctuations of Solvent Around Solute. J. Electroanalytical Chem., 1997, 438, No. 1-2, 245.

54. Chong S.-H., Hirata F. Interaction-Site-Model Description of Collective Excitations in Classical Molecular Fluids. Phys. Rev. E, 1998, 57, No. 2, 1691.

55. Sato H., Hirata F., Myers A.B. Theoretical Study of the Solvent Effect on Triiodide Ion in Solutions. J. Phys. Chem., 1998, 102, No. 11, 2065.

56. Sato H., Hirata F. Theoretical Study for Autoionization of Liquid Water: Temperature Dependence of the Ionic Product (pK(w)). J. Phys. Chem., 1998, A102, No. 15, 2603.

57. Akiyama R., Hirata F. Theoretical Study for Water Structure at Highly Ordered Surface: Effect of Surface Structure. J. Chem. Phys., 1998, 108, No 12, 4904.

58. Chong S.-H., Hirata F. Dynamics of Solvated Ion in Polar Liquids: An Interaction-Site-Model Description. J. Chem. Phys., 1998, 108, No. 17, 7339.

59. Ishida T., Hirata F., Sato H., Kato S. Molecular Theory of Solvent Effect on Keto-Enol Tautomers of Formamide in Aprotic Solvents: RISM-SCF Approach. J. Phys. Chem B, 1998, 102, No. 11, 2045.

60. Kinoshita M., Okamoto Y., Hirata F. First-principle Determination of Peptide Conformations in Solvents: Combination of Monte Carlo Simulated Annealing and RISM Theory. J. Am. Chem. Soc., 1998, 120, No. 8, 1855.

61. Hirata F., Imai T., Irisa M. Molecular Theories of Partial Molar Volume. Rev. High Press. Sci. and Tech., 1998, 8, 96.

62. Kovalenko A., Hirata F. Three-Dimensional Density Profiles of Water in Contact with a Solute of Arbitraly Shape: a RISM Approach, Chem. Phys. Letter, 1998, 290, No. 1-3, 237.

63. Hirata F. Chemical Processes in Solution Studied by an Integral Equation Theory of Molecular Liquids. Bull. Chem. Soc. Jpn., 1998, 71, No. 7, 1483.

64. Chong S.-H., Hirata F. Interaction-Site Representation for Collective Excitations in Diatomic Dipolar Liquid. J. Mol. Liquids, 1998, 77, No. 1-3, 105.

65. Kinoshita M., Okamoto Y., Hirata F. Calculation of Solvation Free Energy Using RISM Theory for Peptide in Salt Solution. J. Comp. Chem., 1998, 19, No. 15, 1724.

66. Kinoshita M., Okamoto Y., Hirata F. Singular Behavior of the Reference Interaction Site Model Theory Observed for Peptide in Salt Solution. Chem. Phys. Letters., 1998, 297, No. 5-6, 433.

67. Chong S.-H., Hirata F. Effect of Molecular Symmetry on Electrical Potential Fluctuations of Solvent Around Solute in Polar Liquid. Chem. Phys. Lett., 1998, 293, No. 1-3, 119.

68. Chong S.-H., Hirata F. Mode-Coupling Theory for Molecular Liquids Based on the Interaction-Site Model. Phys.Rev. E., 1998, 58 No. 5, 6188-6189. 
69. Chong S.-H., Hirata F. Time-Correlation Functions in Molecular Liquids Studied by the ModeCoupling Theory Based on the Interaction-Site Model. Phys. Rev. E, 1998, 58, No. 6, 7296-7308.

70. Ishida T., Hirata F., Kato S. Thermodynamics Analysis of the Solvent Effect on Tautomerization of Acetylacetone: An Ab initio Approach. J. Phys. Chem., 1999, 110 No. 8, 3938-3945.

71. Naka K., Sato H., Morita A., Hirata F., Kato S., RISM-SCF Study for the Free Energy Profile of Menshutkin Type Reaction NH3 + CH3Cl ¿NH3CH3+ + Cl- in Aqueous Solution. Theor. Chem. Acc., 1999, 102, No. 1-6, 165-169.

72. Sato H., Hirata F. The Syn- / Anti- Conformational Equilibrium of Acetic Acid in Water Studied by the RISM-SCF/MCSCF method. THEOCHEM (Morokuma issue), 1999, 461, 113-120.

73. Kovalenko A., Ten-no S., Hirata F. Solution of Three-Dimensional Reference Interaction Site Model and Hypernetted Chain Equations for Simple Point Charge Water by Modified Method of Direct Inversion in Iterative Subspace. J. Comp. Chem., 1999, 20, No. 19, 928-936.

74. Kinoshita M., Okamoto Y., Hirata F. Analysis on Conformational Stability of C-peptide of Ribonuclease A in Water Using the Reference Interaction Site Model Theory and Monte Carlo Simulated Annealing. J. Chem. Phys., 1999, 110, No. 8, 4090-4100.

75. Sato H., Hirata F. Revisiting the Acid-Base Equilibrium in Aqueous Solutions of Hydrogen Halides: Study by the Ab inition Electronic Structure Theory Combined with the Statistical Mechanics of Molecular Liquids. J. Am. Chem. Soc., 1999, 121, No. 14, 3460-3467.

76. Kovalenko A., Hirata F. Self-Consistent Description of a Metal-Water Interface by the Khon-Sham Density Functional Theory and the Three-Dimensional Reference Interaction Site Model. J. Chem. Phys., 1999, 110, No. 20, 10095-10112.

77. Sethia A., Hirata F., Singh Y. Density Matrix for an Excess Electron in a Classical Fluid. Results for a One Dimensional System. J. Chem. Phys., 1999, 110, No. 20, 10086-10094.

78. Hirata F., Chong S.-H. Comment on 'Dynamics of Solvated Ion in Polar Liquids: An InteractionSite-Model Description'-Response. J. Chem. Phys., 1999, 110, No. 3, 1835-1836.

79. Chong S.-H., Hirata F. Dynamics of Ions in Liquid Water: An Interaction-Site-Model Description. J. Chem. Phys., 1999, 111, No. 8, 3654-3667.

80. Chong S.-H., Hirata F. Interaction-Site-Model Descritption of Collective Excitations in Liquid Water I: Theoretical Study. J. Chem. Phys., 1999, 111, No. 7, 3083-3094.

81. Chong S.-H., Hirata F. Interaction-Site-Model Descritption of Collective Excitations in Liquid Water II: Comparison with Simulation Results. J. Chem. Phys., 1999, 111, No. 7, 3095- 3104.

82. Sato H., Hirata F. Ab Initio Study on Molecular and Thermodynamic Properties of Water: A Theoretical Prediction of pKw over a Wide Range of Temperature and Density. J. Phys. Chem. B., 1999, 103, No. 31, 6596-6604.

83. Ishida T., Hirata F., Kato S. Solvation Dynamics of Benzonitrile Excited State in Polar Solvents: A Time-Dependent Reference Interaction Site Model Self-Consistent Field Approach. J. Chem. Phys., 1999, 110, No. 23, 11423-11432.

84. Akiyama R., Kinoshita M., Hirata F. Free Energy Profiles of Electron Transfer at Water-Electrode Interface Studied by the Reference Interaction Site Model Theory. Chem. Phys. Lett., 1999, 305, No. 3-4, 251-257.

85. Mitsutake A., Irisa M., Okamoto Y., Hirata F. Classification of Low-Energy Conformations of MetEnkephalin in the Gas Phase and in a Model Solvent Based on the Extended Scaled Particle Theory. Bull. Chem. Soc. Jpn., 1999, 72, No. 8, 1717-1729.

86. Sato H., Hirata F. Ab Initio Study of Water (II): Liquid Structure, Electronic and Thermodynamic Properties over a wide Range of Temperature and Density. J. Chem. Phys., 1999, 111, No. 18, $8545-8555$.

87. Kovalenko A., Hirata F. Potential of Mean Force between Two Molecular Ions in a Polar Molecular Solvent: a Study by the Three-Dimensional Reference Interaction Site Model. J. Phys. Chem. B, 1999, 103, No. 37, 7942-7957.

88. Sethia A., Hirata F., Tanimura Y., Singh Y. Polaron Density Matrix and Effective Mass at Finite Temperature, Phys. Rev. B, 1999, 60, No. 10, 7245-7251. 
89. Sethia A., Sanyal S., Hirata F. Eigenstates from the Discretized Path Integral. Chem. Phys. Lett., 1999, 315, No. 3-4, 299-303.

90. Harano Y., Sato H., Hirata F. Solvent Effects on a Diels-Alder Reaction in Supercritical Water: RISM-SCF Study. J. Am. Chem. Soc., 2000, 122, No. 10, 2289-2293.

91. Kinoshita M., Okamoto Y., Hirata F. Peptide Conformations in Alcohol and Water: Analysis by the Reference Interaction Site Model Theory. J. Am. Chem. Soc., 2000, 122, No. 12, 2773-2779.

92. Imai T., Kinoshita M., Hirata F. Salt Effect on Stability and Solvation Structure of Peptide: An Integral Equation Study. Bull. Chem. Soc. Jpn., 2000, 73 No. 5, 1113-1122.

93. Kovalenko A., Hirata F. Potentials of Mean Force of Simple Ions in Ambient Aqueous Solution. I. Three-Dimensional Reference Interaction Site Model Approach. J. Chem. Phys., 2000, 112, No. 23, 10391 - 10402.

94. Kovalenko A., Hirata F. Potentials of Mean Force of Simple Ions in Ambient Aqueous Solution. II. Solvation Structure from the Three-Dimensional Reference Interaction Site Model Approach, and Comparison with Simulations. Chem. Phys., 2000, 112, No. 23, 10403-10417.

95. Kovalenko A., Hirata F. Hydration Free Energy of Hydrophobic Solutes Studied by a Reference Interaction Site Model with a Repulsive Bridge Correction and a Thermodynamic Perturbation Method. J. Chem. Phys., 2000, 113, No. 7, 2793-2805.

96. Harano Y., Sato H., Hirata F. Theoretical Study on Diels-Alder Reaction in Ambient and Supercritical Water: Viewing Solvent Effect through the Frontier Orbitals. Chem. Phys., 2000, 258, No. 2-3, $151-161$.

97. Sato H., Kovalenko A., Hirata F. Self-Consistent Field, Ab Initio Molecular Orbital and ThreeDimensional Reference Interaction Site Model Study for Solvation Effect on Carbon Monoxide in Aqueous Solution. J. Chem. Phys., 2000, 112, No. 21, 9463-9468.

98. Imai T., Kinoshita M., Hirata F. Theoretical Study for Partial Molar Volume of Amino Acids in Aqueous Solution: Implication of Ideal Fluctuation Volume. J. Chem. Phys., 2000, 112, No. 21, 9469-9478.

99. Shapovalov V., Truong T. N., Kovalenko A., Hirata F., Liquid Structure at Metal Oxide-Water Interface: Accuracy of a Three-Dimensional RISM Methodology. Chem. Phys. Lett., 2000, 320, No. 1-2, 186-193.

100. Sato H., Matsubayashi N., Nakahara M., Hirata F. Which Carbon Oxide is More Soluble? Ab Initio Study on Carbon Monoxide and Dioxide in Aqueous Solution. Chem. Phys. Lett., 2000, 323, No. 3-4, $257-262$.

101. Sethia A., Hirata F. Electron Self-Trapping in Two-Dimensional Fluid. Chem. Phys. Lett., 2000, 326, No. 3-4, 199-206.

102. Yamazaki T., Sato H., Hirata F. NMR Chemical Shifts in Solution: A RISM-SCF Approach. Chem. Phys. Lett., 2000, 325, No. 5-6, 668-674.

103. Kovalenko A., Hirata F., Kinoshita M. Hydration Structure and Stability of Met-Enkephalin Studied by a Three-Dimensional Reference Interaction Site Model with a Repulsive Bridge Correction and Thermodynamic Perturbation Method. J. Chem. Phys., 2000, 113, No. 21, 9830-9836.

104. Nishiyama K., Hirata F., Okada T. Importance of Acoustic Solvent Mode and Solute-Solvent Radial Distribution Functions in Slvation Dynamics: Studied by RISM Theory. J. Chin. Chem. Soc., 2000, 47, No. 4A, 837-842.

105. Nishiyama K., Hirata F., Okada T. Relaxation of Average Energy and Rearrangement of Solvent Shells in Various Polar Solvents in Connection with Solvation Dynamics: Studied by RISM Theory. Chem. Phys. Lett., 2000, 330, No. 1-2, 125-131.

106. Mitsutake A., Kinoshita M., Okamoto Y., Hirata F., Multicanonical Algorithm Combined with the RISM Theory for Simulating Peptide in Aqueous Solution. Chem. Phys. Lett., 2000, 329, No. 3-4, 295-303.

107. Nakabayashi T., Sato H., Hirata F., Nishi N. Theoretical Study on the Structures and Energies of Acetic Acid Dimers in Aqueous Solution. J. Phys. Chem. A, 2001, 105, No. 1, 245-250.

108. Sethia A., Sanyal S., Hirata F., Quantum Dynamics: Path Integral Approach to Time Correlation Functions in Finite Temperature. J. Chem. Phys., 2001, 114, No. 11, 5097-5098. 
109. Sethia A., Miura S., Hirata F. Density Matrix and Eigenstates for an Excess Electron in Water. J. Mol. Liquids, 2001, 90, No. 1-3, 225-231.

110. Kinoshita M., Okamoto Y., Hirata F. Solvent Effects on Conformational Stability of Peptides: RISM Analyses. J. Mol. Liquids, 2001, 90, No. 1-3, 195-204.

111. Kovalenko A., Hirata F. Self-Consistent, Kohn-Sham DFT and Three-Dimensional RISM Description of a Metal-Molecular Liquid Interface. J. Mol. Liquids, 2001, 90 No. 1-3, 215-224.

112. Nishiyama K., Hirata F., Okada T. Average Energy Relaxation and Rearrangement of Solute-Solvent Radial Distribution Function in Solvation Dynamics: A Connection between Spectroscopic Results and RISM Theory. J. Mol. Liquids, 2001, 90, No. 1-3, 251-260.

113. Harano Y., Imai T., Kovalenko A., Kinoshita M., Hirata F., Theoretical Study for Partial Molar Volume of Amino Acids and Polypeptides by the Three-Dimensional Reference Interaction Site Model. J. Chem. Phys., 2001, 114, No. 21, 9506-9511.

114. Nishiyama K., Hirata F., Okada T. Nonlinear Response of Solvent Molecules Induced by Instantaneous Change of Solute Electronic Structure: Studied by RISM Theory. J. Mol. Struct., 2001, 565, $31-34$.

115. Kovalenko A., Hirata F. A Replica Reference Interaction Site Model Theory for a Polar Molecular Liquid Sorbed in a Disordered Microporous Material with Polar Chemical Groups. J. Chem. Phys., 2001, 115, No. 18, 8620-8633.

116. Sato H., Hirata F. Realization of Three-Dimensinal Solvation-Structure from the Site-Site Radial Distribution Functions in Liquids. Bull. Chem. Soc. Jpn., 2001, 74, No. 10, 1831-1839.

117. Imai T., Harano Y., Kovalenko A., Hirata F. Theoretical Study for Volume Changes Associated with the Helix-Ciol Transition of Peptides. Biopolymers, 2001, 59, No. 7, 512-519.

118. Sumi T., Imai T., Hirata F. Integral Equations for Molecular Fluids Based on Interaction Site Model: Density-Functional Formulation. J. Chem. Phys., 2001, 115, No. 14, 6653-6662.

119. Yamazaki T., Sato H., Hirata F. Solvent Effect on the Nuclear Magnetic Shielding: Ab Initio Study by the Combined Reference Interaction Site Model and Electronic Structure Theories. J. Chem. Phys., 2001, 115, No. 19, 8949-8957.

120. Yamaguchi T., Hirata F. Site-Site Mode-Coupling Theory for the Shear Viscosity of Molecular Liquids. J. Chem. Phys., 2001, 115, No. 20, 9340-9345.

121. Kovalenko A., Hirata F. First-Principles Realization of a Van Der Waals-Maxwell Theory for Water. Chem. Phys. Lett., 2001, 349, No. 5-6, 496-502.

122. Kovalenko A., Hirata F. Description of a Polar Molecular Liquid in a Disordered Microporous Material with activating chemical groups by a replica RISM theory. Cond. Mat. Phys., 2001, 4, 643-678.

123. Nishiyama K., Hirata F., Okada T. Solute-Shape Dependence in Solvation Dynamics: Investigated by RISM Theory. J. Mol. Liquids, 2002, 96, No. 7, 391-395.

124. Kinoshita M., Imai T., Kovalenko A., Hirata F. Improvement of the Reference Interaction Site Model Theory for Calculating the Partial Molar Volume of Amino Acids and Polypeptides. Chem. Phys. Lett., 2001, 348, No. 3-4, 337-342.

125. Yamaguchi T., Hirata F. Translational Diffusion and Reorientational Relaxation of Water Analyzed by Site-Site Generalized Langevin theory. J. Chem. Phys., 2002, 116, No. 6, 2502-2507.

126. Sato H., Hirata F. Equilibrium and Nonequilibrium Solvation Structure of Hexaamineruthenium (II,III) in Aqueous Solution: Ab Initio RISM-SCF Study. J. Phys. Chem. A, 2002, 106, No. 10, 2300-2304.

127. Yoshida K., Kovalenko A., Yamaguchi T., Hirata F. Structure of Tert-Butyl Alcohol-Water Mixtures Studied by the RISM Theory. J. Phys. Chem. B., 2002, 106, No. 19, 5042.

128. Imai T., Nomura H., Kinoshita M., Hirata F. Partial Molar Volumes and Compressibilities of AlkaliHalide Ions in Aqueous Solution: Hydration Shell Analysis with an Integral Equation Theory of Molecular Liquids. J. Phys. Chem. B, 2002, 106, No. 29, 7308- 7314.

129. Yamaguchi T., Hirata F. Interaction-Site Model Description of the Reorientational Relaxation of Molecular Liquids: Incorporation of the Interaxial Coupling into the Site-Site Generalized Langevin / Mode-Coupling Theory. J. Chem. Phys., 2002, 117, No. 5, 2216- 2224. 
130. Kimura T., Matsubayashi N., Sato H., Hirata F., Nakahara M. Enthalpy and Entropy Decomposition of Free-Energy Changes for Side-Chain Conformations of Asparatic Acid and Asparagine in Acidic, Neutral, and Basic Aqueous Solutions. J. Phys. Chem. B, 2002, 106, No. 47, 12336-12343.

131. Sumi T., Hirata F. A Density-Functional Theory for Polymer Liquids Based on Interaction Site Model. J. Chem. Phys., 2003, 118, No. 5, 2431-2442.

132. Solute-Structure Dependence of Solvation Dynamics Studied by Reference Interaction-Site Model Theory. Nishiyama K., Hirata F., Okada T. J. Chem. Phys., 2003, 118, No. 5, 2279- 2285.

133. Yamaguchi T., Chong S.-H., Hirata F. Dielectric Relaxation Spectrum of Water Studied by the Site-Site Generalized Langevin / Modified Mode-Coupling Theory. Mol. Phys., 2003, 101, No. 8, 1211-1220.

134. Yamaguchi T., Chong S.-H., Hirata F. Theoretical Study of the Molecular Motion of Liquid Water under High Pressure. J. Chem. Phys., 2003, 119, No. 2, 1021- 1034.

135. Imai T., Hirata F. Partial Molar Volume and Compressibility of a Molecule with Internal Degrees of Freedom. J. Chem. Phys., 2003, 119, No. 11, 5623- 5631.

136. Sato H., Kobori Y., Tero-Kubota S., Hirata F. Theoretical Study on Electronic and Solvent Reorganization Associated with a Charging Process of Organic Compounds: I. Molecular and Atomic Level Description of Solvent Reorganization. J. Chem. Phys., 2003, 119, No. 5, 2753-2760.

137. Omelyan I., Kovalenko A., Hirata F. Compressibility of Tert-Butyl Alcohol-Water Mixtures: the RISM Theory. J. Theor. Comp. Chem., 2003, 2, No. 2, 193- 203.

138. Interplay between the Repulsive and Attractive Interaction and the Spacial Dimesionality of an Excess Electron in a Simple Fluid, A. Sethia, E. R. Bittner F. Hirata, J. Theor. Comp. Chem., 2003, 2(2), 129-138.

139. Tanimura A., Kovalenko A., Hirata F. Molecular Theory of an Electrochemical Double Layer in a Nanoporous Carbon Supercapacitor. Chem. Phys. Lett., 2003, 378, No. 5-6, 638-646.

140. Yamazaki T., Sato H., Hirata F. A Quantum Solute-Solvent Interaction Using Spectral Representation Technique Applied to the Electronic Structure Theory in Solution. J. Chem. Phys., 2003, 119, No. 13, 6663-6670.

141. Kovalenko A., Hirata F. Molecular Description of Electrolyte Solution in a Carbon Aerogel Electrode. Cond. Matt. Phys., 2003, 6, 583-609.

142. Yamaguchi T., Chong S.-H., Hirata F. Mode-Coupling Analysis of the Translational and Rotational Diffusion of Polar Liquids; Acetonitrile and Water. J. Mol. Liquids, 2004, 112, No. 3, 117-124.

143. Kobori Y., Yago T., Akiyama K., Tero-Kubota S., Sato H., Hirata F., Norris J. R. Superexchange Electron Tunneling Mediated by Solvent Molecules: Pulsed Electron Paramagnetic Resonance Study on Electronic Coupling in Solvent-Separated Radical Ion Pairs. J. Phys. Chem. B, 2004, 108, No. 29, $10226-10240$.

144. Sato H., Hirata F., Sakaki S. Distortion of Electronic Structure in Solvated Molecules: Tautomeric Equilibrium of 2-Pyridone and 2-Hydroxypridine in Water Studied by the RISM-SCF Method. J. Phys. Chem. A, 2004, 108, No. 11, 2097-2102.

145. Sato H., Kobori Y., Tero-Kubota S., Hirata F. Theoretical Study on Electronic and Solvent Reorganization Associated with a Charging Process of Organic Compounds: 2. A New Decomposition Procedure into Electrostatic and Nonelectrostatic Responses. J. Phys. Chem. B, 2004, 108, No. 31, 11709-11715.

146. Imai T., Kovalenko A., Hirata F. Solvation Thermodynamics of Protein Studied by the 3D-RISM Theory. Chem. Phys. Lett., 2004, 395, No. 1-3, 1-6.

147. Mitsutake A., Kinoshita M., Okamoto Y., Hirata F. Combination of the Replica-Exchange Monte Carlo Method and the Reference Interaction Site Model Theory for Simulating a Peptide Molecule in Aqueous Solution. J. Phys. Chem. B, 2004, 108, No. 49, 19002-19012.

148. Nishiyama K., Yamaguchi T., Hirata F., Okada T., Solute Dependence of Polar Solvation Dynamics Studied by RISM/Mode-Coupling Theory. J. Solution Chem., 2004, 33, No. 6-7, 631-639.

149. Omelyan I., Kovalenko A., Hirata F. Microscopic Description of a Liquid-Vapor Interface by an Inhomogeneous Integral Equation Theory. Chem. Phys. Lett., 2004, 397, No. 4-7, 368-373. 
150. Nishimura K., Yamaguchi T., Hirata F., Okada T. Polar Solvention Dynamics: A Combination of the Reference Interaction-Site Model and Model-Coupling Theories. Pure and Applied Chem., 2004, 76, No. (1).

151. Hydrophobic Effects on Partial Molar Volume, T. Imai, F. Hirata, J. Chem. Phys., 2005, 122, No. 9 , Art. No. 094509.

152. Kobryn A.E., Yamaguchi T., Hirata F. Pressure Dependence of Diffusion Coefficient and Orientational Relaxation Time for Acetonitrile and Methanol in Water: RISM/Mode-Coupling Study. J. Mol. Liquids, 2005, 119, No. 1-3, 7-13.

153. Nishiyama K., Yamaguchi T., Hirata F., Okada T., Solvation Dynamics in Water Investigated by RISM/Mode-Coupling Theory. J. Mol. Liquid, 2005, 119, No. 1-3, 63-66.

154. Imai T., Kovalenko A., Hirata F. Partial Molar Volume of Proteins Studied by the Three-Dimensional Reference Interaction Site Model Theory. J. Phys. Chem. B, 2005, 109, No. 14, 6658-6665.

155. Kobryn A.E., Yamaguchi T., Hirata F. Site-Site Memory Equation Approach in Study of Density / Pressure Dependence of Translational Diffusion Coefficient and Rotational Relaxation Time of Polar Molecular Solutions: Acetonitrile in Water, Methanol in Water, and Methanol in Acetonitrile. J. Chem. Phys., 2005, 122, No. 18, 184511-184524.

156. Kovalenko A., Hirata F. A Molecular Theory of Liquid Interfaces. Phys. Chem. Chem. Phys., 2005, 7, No. 8, 1785-1793.

157. Imai T., Takahiro T., Kovalenko A., Hirata F., Kato M., Taniguchi Y. Theoretical Study of Vomule Changes Associated with the Helix-Coil Transition of an Alanine-Rich Peptide in Aqueous Solution. Biopolymers, 2005, 79, No. 2, 97-105.

158. Imai T., Hiraoka R., Kovalenko A., Hirata F., Water Molecules in a Protein Cavity Detected by a Statistical-Mechanical Theory. J. Am. Chem. Soc., 2005, 127, No. 44, 15334-15335.

159. Omelyan I., Hirata F., Kovalenko A. Criticality of a Liquid-Vapor Interface from an Inhomogeneous Integral Equation Theory. Phys. Chem. Chem. Phys., 2005, 7, No. 24, 4132-4137.

160. Reply to "Comment on 'Hydrophobic Effects on Partial Molar Volume", [ J. Chem. Phys., 2005, 123, 167103] Imai T., Hirata F., J. Chem. Phys., 2005, 123, No. 16, Art. No. 167104.

161. Ishizuka R., Hirata F. Conformational Equilibria in Liquids Consisting of Small Chain Molecules. Chem. Phys. Lett., 2006, 420, No. 1-3, 135-139.

162. Yoshida N., Hirata F. A New Method to Determine Electrostatic Potential Around a Macromolecule in Solution from Molecular Wave Functions. J. Comput. Chem., 2006, 27, No. 4, 453-462.

163. Yoshida N., Ishizuka R., Sato H., Hirata F. Ab Initio Theoretical Study of Temperature and Density Dependence of Molecular and Thermodynamic Properties of Water in the Entire Fluid Region: Autoionization Processes. J. Phys. Chem. B, 2006, 110, No. 16, 8451-8458.

164. Kobryn A.E., Yamaguchi T., Hirata F. Study of Anomalous Mobility of Polar Molecular Solutions by Means of the Site-Site Memory Equation Formalism. J. Mol. Liquids, 2006, 125, No. 1, 14-21.

165. Imai T., Isogai H., Seto T., Kovalenko A., Hirata F. Theoretical Study of Volume Changes Accompanying Xenon-Lysozyme Binding: Implications for Molecular Mechanism of Pressure Reversal of Anesthesia. J. Phys. Chem. B, 2006, 110, No. 24, 12149-12154.

166. Imai T., Harano Y., Kinoshita M., Kovalenko A., Hirata F., A Theoretical Analysis on Hydration Thermodynamics of Proteins. J. Chem. Phys., 2006, 125, No. 2, 024911.

167. Lee J.Y., Yoshida N., Hirata F. Conformational Equilibrium of 1,2-Dichloroethane in Water: Comparison of PCM and RISM-SCF Methods. J. Phys. Chem. B, 2006, 110, No. 32, 16018-16025.

168. Yoshida N., Phongphanphanee S., Maruyama Y., Imai T., Hirata F. Selective Ion-Binding by Protein Probed with the 3D-RISM Theory. J. Am. Chem. Soc., 2006, 128, No. 37, 12042 -12043.

169. Kobryn A.E., Hirata F. Statistical-Mechanical Theory of Ultrasonic Absorption in Molecular Liquids. J. Chem. Phys., 2007, 126, No. 4, Art. No. 044504.

170. Imai T., Hiraoka R., Kovalenko A., Hirata F. Locating Missing Water Molecules in Protein Cavities by the Three- Dimensional Reference Interaction Site Model Theory of Molecular Solvation. ProteinsStructure Function and Bioinformatics, 2007, 66, No. 4, 804-813.

171. Ikuta Y., Maruyama Y., Matsugami M., Hirata F. Probing Cations Recognized by a Crown Ether with the 3D-RISM Theory. Chem. Phys. Lett., 2007, 433, No. 4-6, 403-408. 
172. Tanimura A., Kovalenko A., Hirata F. Structure of Electrolyte Solutions Sorbed in Carbon Nanospaces, Studied by the Replica RISM Theory. Langmuir, 2007, 23, No. 3, 1507-1517.

173. Yamazaki T., Imai T., Hirata F., Kovalenko A. Theoretical Study of the Cosolvent Effect on the Pratial Molar Volume Change of Staphylococcal Nuclease Associated with Pressure Denaturation. J. Phys. Chem. B, 2007, 111, No. 5, 1206-1212.

174. Yoshida N., Phonphanphanee S., Hirata F. Selective Ion-Binding by Protein Probed with the Statistical Mechanical Integral Equation Theory. J. Phys. Chem. B, 2007, 111, No. 17, 4588-4595.

175. Ikuta Y., Maruyama Y., Hirata F., Tomoda S., Theoretical Study of Solvent Effect on Diastereo Selectivity in Protonation of Methyl 3-Fluorobutanoate Anion by Ethanol: Application of the 3DRISM Theory. J. Mol. Struct.-Theochem, 2007, 811, No. 1-3, 183-190.

176. Imai T., Ohyama S., Kovalenko A., Hirata F., Theoretical study of the partial molar volume change associated with pressure-induced structural transition of ubiquitin, Protein Science, 2007, 16, 19271933.

177. Imai T., Harano H., Kinoshita M., Kovalenko A., A theoretical analysis on changes in thermodynamic quantities upon protein folding: Essential role of hydration, J. Chem. Phys., 2007, 126, 225102225102 . 